\title{
Genome-wide identification and characterization of SnRK family genes in Brassica napus
}

\author{
Weizhuo Zhu, Dezhi Wu, Lixi Jiang and Lingzhen Ye*
}

\begin{abstract}
Background: Sucrose non-fermenting 1 related protein kinases (SnRK) play crucial roles in responding to biotic and abiotic stresses through activating protein phosphorylation pathways. However, little information of SnRK genes was available in Brassica napus, one of important oil crops. Recently, the released sequences of the reference genome of B.napus provide a good chance to perform genome-wide identification and characterization of BnSnRK gene family in the rapeseed.

Results: Totally 114 SnRK genes distributed on 19 chromosomes were identified in the genome of B.napus and classified into three subfamilies on the basis of phylogenetic analysis and the domain types. According to gene structure and motif composition analysis, the BnSnRK sequences showed obvious divergence among three subfamilies. Gene duplication and synteny between the genomes of the rapeseed and Arabidopsis were also analyzed to provide insights into the evolutionary characteristics of BnSnRK family genes. Cis-element analysis revealed that BnSnRKs may response to diverse environmental stresses. Moreover, the expression patterns of BnSnRKs in various tissues and under diverse abiotic stresses were distinct difference. Besides, Single Nucleotide Polymorphisms (SNP) distribution analysis suggests the function disparity of BnSnRK family genes in different genotypes of the rapeseed.
\end{abstract}

Conclusion: We examined genomic structures, evolution features, expression patterns and SNP distribution of 114 BnSnRKs. The results provide valuable information for functional characterization of BnSnRK genes in future studies.

Keywords: Brassica napus, SnRK, Genome-wide, Abiotic stress, Expression patterns

\section{Background}

Plants develop various molecular defense mechanisms to cope with abiotic stresses, including salinity, drought and cold stresses. Gene expression regulation and protein modification are two important ways for plants to deal with these stresses [1]. The processes of phosphorylation and dephosphorylation mediated by protein kinase play crucial roles in protein modification [2]. Among the reported protein kinase genes, sucrose non-

\footnotetext{
* Correspondence: yelingzhen@zju.edu.cn

Institute of Crop Science, Zhejiang University, Hangzhou 310058, China
}

fermenting 1 (SNF1)-related protein kinases $(S n R K s)$ are involved in different physiological processes [3].

In plants, SnRKs could be divided into three subfamilies: $\operatorname{SnRK1}$, SnRK2 and SnRK3 based on their sequence similarity and gene structures [3, 4]. In detail, the SnRK1 subfamily contain a highly conserved $\mathrm{N}$-terminal protein kinase (Pkinase) domain, which are the homologous genes of SNF1 in yeasts and AMPKS in mammals [5]. The other subfamilies $S n R K 2 / 3$ are unique in plants, both are more diverse in plants than the SnRK1 subfamily members. The member of $S n R K 2$ harbors a conserved Pkinase domain and a C-terminal variable adjusting conserved domain [6]. In addition, SnRK3

(c) The Author(s). 2020 Open Access This article is licensed under a Creative Commons Attribution 4.0 International License, which permits use, sharing, adaptation, distribution and reproduction in any medium or format, as long as you give appropriate credit to the original author(s) and the source, provide a link to the Creative Commons licence, and indicate if changes were made. The images or other third party material in this article are included in the article's Creative Commons licence, unless indicated otherwise in a credit line to the material. If material is not included in the article's Creative Commons licence and your intended use is not permitted by statutory regulation or exceeds the permitted use, you will need to obtain permission directly from the copyright holder. To view a copy of this licence, visit http://creativecommons.org/licenses/by/4.0/ The Creative Commons Public Domain Dedication waiver (http://creativecommons.org/publicdomain/zero/1.0/) applies to the data made available in this article, unless otherwise stated in a credit line to the data. 
known as CIPK (CBL-interacting protein kinases), also contain conserved $\mathrm{N}$-terminal protein kinase domains and NAF domains, PPI domains in C-terminal $[7,8]$.

The SnRK1 family genes involve in the response of plant cells to starvation and metabolic stress. SnRK1 kinases are the catalytic subunits of heterotrimeric complexes that interact with two other subunits [9]. In Solanum tuberosum, SnRK1 was proved to be involved in induction of sucrose synthase expression and played an important role in carbohydrate metabolism regulation [10]. Besides, low energy stress (e.g. darkness and hypoxia) could trigger $S n R K 1 \alpha$ nuclear translocation and further induce SnRK1 target genes to refresh cellular energy for plant growth [11]. Furthermore, considerable evidences indicated that SnRK1 genes were hubs in a network of various signaling pathways including cell cycle regulation, pathogen responses and meristem development [12].

On the other hand, the SnRK2 genes play important roles in responding to abiotic stresses in plants, especially for osmotic and salt stress. For instance, SnRK2.10 phosphorylate several target genes including dehydrins ERD10 and ERD14 to deal with osmotic stress in Arabidopsis thaliana [13]. SnRK2.1 positivly regulate salt tolerance in Nicotiana tabacum [14, 15]. In A. thaliana, SnRK2 subfamily genes could be classified into three groups: group 1 contains the ABA-independent kinases, group 2 includes genes responding to drought stress, and group 3 kinases are strongly stimulated by ABA $[6,16]$. The current researches on ABA-dependent group 3 kinases are the most extensive. For example, AtSnRK2.6 (OST1), one of the SnRK2 family gene, plays a core role in the ABA signaling pathway in stomatal guard cells, and OST1 protein stability can be regulated via E3-ubiquitin ligase HOS15 to reduce ABA signal sensitivity in Arabidopsis [17, 18].

SnRK3 kinases known as CIPKS (CBL (calcium sensor calcineurin B-like proteins)-interacting protein kinases), perform vital functions in resistance to various stresses in plants [3, 19]. For example, SOS (salt overly sensitive) system was the first discoverd $C B L-C I P K$ pathway in A.thaliana. In detail, the calcium signal produced by salt stress was sensed by SOS3 (AtCBL4) on cell membrane, and then SOS3 combined with SOS2 (AtCIPK24) forming complex to phosphorylate SOS1 $\left(\mathrm{Na}^{+} / \mathrm{H}^{+}\right.$antiporter $)$to remove excess $\mathrm{Na}^{+}$out of root cells $[20,21]$. Besides, MdCIPK13 and MdCIPK22 enhanced salt and drought tolerance through targeting sucrose transporter MdSUT2.2 for phosphorylation in apple [22, 23]. Overexpression of BnCBL1-BnCIPK6 in B.napus could enhance high salinity tolerance and low potassium tolerance [24]. In conclusion, increasing evidences emphasized the importance of SnRKs function in nutrition utilization and stress response, and finally researchers could improve plants resistance to stresses by genetic manipulation using these genes.

Brassica napus is an important oil crop in the world. Recently, the genomes of Darmor-bzh (winter ecotype), Zhongshuang 11 and NY7 (seni-winter ecotype) were successfully sequenced and assembled [25-27]; however, the systematic analysis of $B n S n R K$ gene family has been not well reported. In this study, $114 \operatorname{SnRK}$ gene members were identified in the B.napus genome. We systematically analyzed their phylogenetic relationships, protein motifs, gene structures, chromosome distributions and cis-elements in promoter regions. Moreover, the expression profiles of $B n S n R K s$ in diverse tissues as well as under abiotic stresses were determined. In addition, SNPs of each BnSnRK gene were systematically identified in a worldwide collection with 300 core germplasm accessions. These results will provide useful information for further investigation of molecular mechanisms of $B n S n R K$ genes for abiotic stress tolerance and molecular breeding in B.napus.

\section{Results \\ Identification and phylogenetic analysis of SnRK genes in B.napus}

A total of 114 proteins with Ser/Thr kinase domain were identified as the members of SnRK family in the B.napus genome (Table S2). The longest amino acid sequence of each protein was selected for further analyses. The information of gene names, ID, chromosomal locations, amino acid numbers, molecular weights (MW), isoelectric points (pI) and domains were listed in Tables S1 and S2. The amino acid length of 114 BnSnRKs protein is ranged from 190 to 1241 , correspondingly the melocular weight ranges from 22.0 to $140.6 \mathrm{kDa}$. The coding regions and sequences of each gene were listed in Table S3.

To analyze evolutionary relationships of $\operatorname{SnRK}$ genes in B.napus and A.thaliana, an unrooted phylogenetic tree was constructed using the full-length amino acid sequences of all SnRKs. Totally, 39 SnRKs from A.thaliana and 114 SnRKs from B.napus were identified and used in the study (Fig. 1). It was reported that 39 AtSnRKs could be clustered into three groups [3]. In this study, based on the phylogenetic analysis, $114 \mathrm{BnSnRKs}$ were also classfied into three groups. In details, 10 proteins are in $B n S n R K 1$ subfamily with Pkinase (PF00069 of Pfam), UBA (PF00627) and KA1 (PF02149) domains, excepting $B n S n R K 1.3$, while 31 proteins contain pkinase domain in $B n S n R K 2$ subfamily with high similarity to AtSnRK2 subfamily, and 73 proteins with Pkinase and NAF (PF03822) domains are in SnRK3 subfamily, respectively (Fig. 1).

\section{Gene structure and protein motif analysis of BnSnRK genes}

The web server GSDS (Gene Structure Display Server) analysis was done to determine gene structures of 


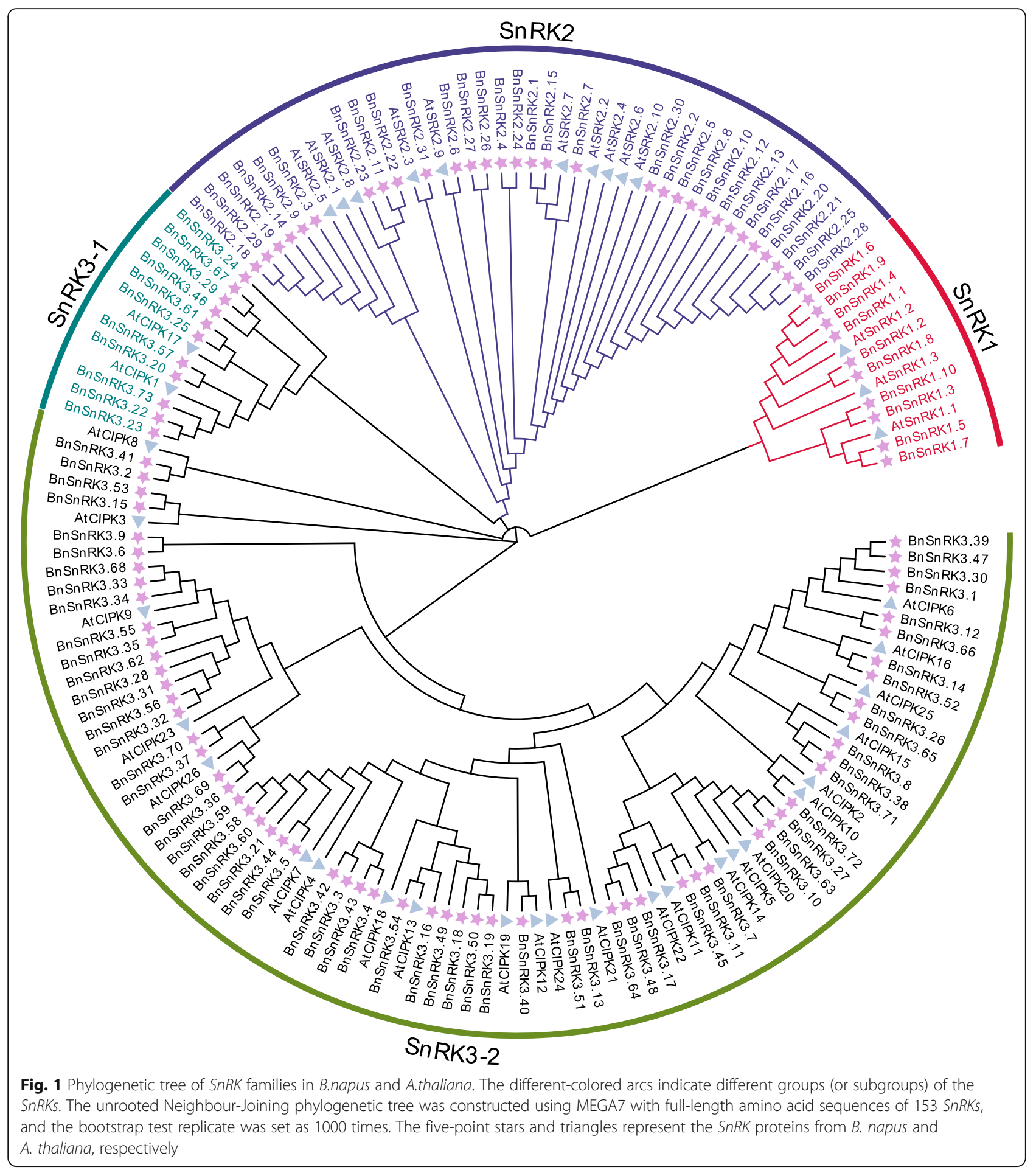

$B n S n R K$ s. As shown in Fig. 2, one to fifteen exons were found in these $B n S n R K$ genes. The BnSnRK1 subfamily genes have more than 6 exons, and 6 to 13 exons for the $\mathrm{BnSnRK2}$ subfamily genes. While it is various for the $B n S n R K 3$ subfamily with ranged from 1 to 15 exons. Furthermore, there are two subgroups in the $B n S n R K 3$ family. The genes in the $S n R K 3$ subgroup 1 contained more than 8 exons, while the genes in the subgroup 2 contain less than 4 exons.

The schematic structures of all $B n S n R K$ proteins were constructed using the MEME (Multiple Em for Motif Elicitation) motif analysis results (Fig. 2c). The sequence and length information of the conserved motif were shown in Supplementary Table S4. In this study, it was 


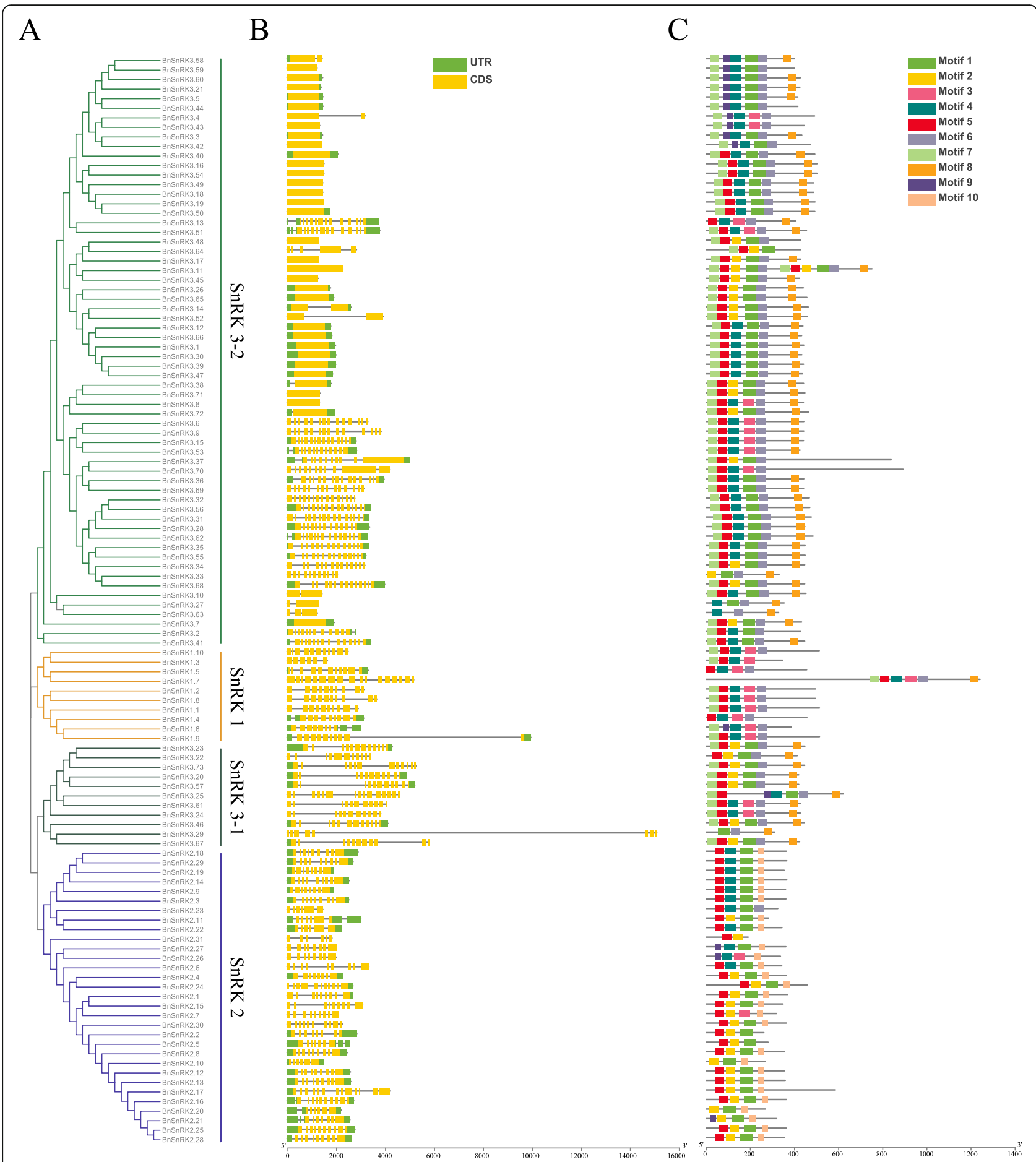

Fig. 2 Phylogenetic relationships, architecture of conserved protein motifs and gene structure of the SnRK genes from B.napus. a Phylogenetic tree of 104 BnSnRK proteins. The unrooted neighbor-joining phylogenetic tree was constructed with MEGA7 using full-length amino acid sequences of 104 BnSnRK proteins, and the bootstrap test replicate was set as 1000 times. $\mathbf{b}$ Exon/intron organization of BnSnRK genes. Yellow boxes represent exons and black lines with same length represent introns. The upstream/downstream region of BnSnRK genes are indicated in green boxes. The length of exons can be inferred by the scale at the bottom. $\mathbf{c}$ The motif composition of BnSnRK proteins. The motifs, numbers 1-10, are displayed in different colored boxes. The sequence information for each motif is provided in Table 54 . The length of protein can be estimated using the scale at the bottom 
found that all $B n S n R K$ genes retained the conserved Pkinase domain containing the motif 1, 2, 3, 4, 5 (Fig. 2c). Moreover, $B n S n R K$ genes within the same subfamily share a similar motif composition, while $B n S n R K$ genes between diverse subfamilies showed distinct differences in motif composition. In detail, $B n S n R K 1$ subfamily genes contain motif $3,4,5,6,7 ; B n S n R K 2$ genes have motif $1,2,5,6,10$ or motif $1,4,5,6,10$; BnSnRK3-1 genes contain motif $1,2,5,6,7,8$ or motif $1,4,5,6,7$, 8; BnSnRK3-2 genes have motif $1,5,6,7,8$ or motif 1 , 6, 7, 8, 9 (Fig. 2c). In summary, the conserved motif compositions and similar gene structures of the SnRK genes within the same subfamily, strongly support the reliability of the subfamily classifications by phylogenetic analysis.

\section{Chromosomal distribution, genome synteny and gene duplication of BnSnRK genes}

Chromosomal analysis showed that $110 \mathrm{BnSnRK}$ genes were distributed over 19 chromosomes, excepting 4 genes (BnSnRK1.10, BnSnRK2.30, BnSnRK2.31, $B n S n R K 3.73)$ could not be mapped into chromosomes of the rapeseed genome (Fig. 3). Among them, 56 $B n S n R K$ genes were located in the AA subgenome, including 4 BnSnRK1 genes, 14 BnSnRK2 genes, 38 $B n S n R K 3$ genes; while 54 genes were located in the CC subgenome, containing 5 BnSnRK1genes, 15 BnSnRK2 genes, 34 BnSnRK3 genes. In addition, some BnSnRK2 and $B n S n R K 3$ subfamily genes were formed as clusters in diverse chromosomes, such as BnSnRK3.22 and 3.23 (Fig. 3). These results indicated that $B n S n R K$ genes were randomly distributed in the chromosomes of the AA or CC subgenome.

Using BLAST and MCScanX methods, 106 segmental duplication events were identified (Fig. 4 and Table S5). Among these events, 104 events were happened between diverse chromosomes, while only 2 duplication events were detected within same chromosome. The result suggested that part of $B n S n R K$ genes were possibly generated by gene duplication, and the segmental duplication events played vital roles in the expansion of $S n R K$ genes in the B.napus genome. We also analyzed the occurrence of the tandem duplication events. Here, 25 tightly linked BnSnRK genes (BnSnRK2.7/2.8, BnSnRK3.3/3.4, BnSnRK3.18/3.19, BnSnRK3.22/3.23, BnSnRK3.24/3.25, BnSnRK3.33/3.34, BnSnRK3.36/3.37, BnSnRK3.42/3.43, BnSnRK3.49/3.50, BnSnRK3.58/3.59/3.60, BnSnRK3.69/ 3.70, BnSnRK3.71/3.72) were found located less than $200 \mathrm{~kb}$. However, the identities of these gene pairs were less than $70 \%$, indicating they were not tandem duplication events.

Futhermore, the synteny of SnRK gene pairs between B.napus genome and A.thaliana genome was performed. The result showed that $65 \mathrm{SnRK}$ genes of
B.napus exhibiting syntenic relationship with AtSnRK genes (Fig. 5 and Table S6), suggesting that these genes might have contributed to the evolution of $B n S n R K$ gene family. To test the evolutionary constraints acting, $K s$ values, $K a$ values, $\mathrm{Ka} / \mathrm{Ks}$ ratios and divergence time of paralogous and orthologous on SnRK family genes were calculated (Tables S5 and S6). The majority of segmental duplicated $B n S n R K$ gene pairs had $K a / K s$ ratios less than 1 , and the mean value of $B n S n R K 3$ gene pairs $(K a / K s=0.30)$ was lower than BnSnRK1 $(K a / K s=0.41)$ and $B n S n R K 2(K a / K s=$ $0.75)$. In addition, the $K a / K s$ ratios of most orthologous $S n R K$ gene pairs were less than 1, and the mean value of $S n R K 2$ gene pairs $(K a / K s=0.09)$ was much lower than that of SnRK1 $(K a / K s=0.75)$ and SnRK3 $(K a / K s=0.23)$. These results suggested that the $B n S n R K$ gene family may have suffered robust purifying selective pressure during evolution.

\section{Stress-related cis-elements in the promoters of BnSnRK genes}

To understand the potential function and regulatory mechanisms of BnSnRK genes, cis-elements (1.5-kb upstream from ATG) were analyzed by using PlantCARE. Totally 104 of 114 BnSnRKs were identified with cis-elements including DRE, ABRE and LTRE, involving in dehydration responses, $\mathrm{ABA}$ responses and low-temperature responses (Fig. 6, Table S2). In detail, 88 BnSnRK genes (77.19\%) had ABRE cis-elements, 33 BnSnRK genes (28.95\%) carried DRE cis-elements, and 44 BnSnRK genes (38.60\%) owned LTRE cis-elements. It was also found that most genes had more than one kind of cis-element types. Furthermore, the number of cis-elements in BnSnRK1 (1.80) family was higher than BnSnRK2 (1.17) and BnSnRK3 (1.21) families (Table S2). In conclusion, the cis-elements analysis suggested that most BnSnRK genes could be responsed to diverse environmental stresses, and different subfamily genes may be regulated diversely.

\section{Expression profiles of BnSnRKs in different tissues}

The expression profiles of $114 \mathrm{BnSnRK}$ genes were compared among 12 tissues of the rapeseed cultivar ZS11 (Fig. 7 and Table S7). The expression pattern of BnSnRKs was divided into three groups. A total of 67 genes with high expression level in all examined tissues ( $\log 2$-based values $>6$ ) were classified into the group 1 . For example, BnSnRK3.40 was highly expressed in all vegetative organs with average $\log 2$-based values of 10.85. $21 \mathrm{BnSnRK}$ genes belonging to group2 exhibited relatively low expression levels across the detected tissues (log2-based values $>2$ ). The group3 included 26 $B n S n R K$ genes, with lowest expression levels in all 


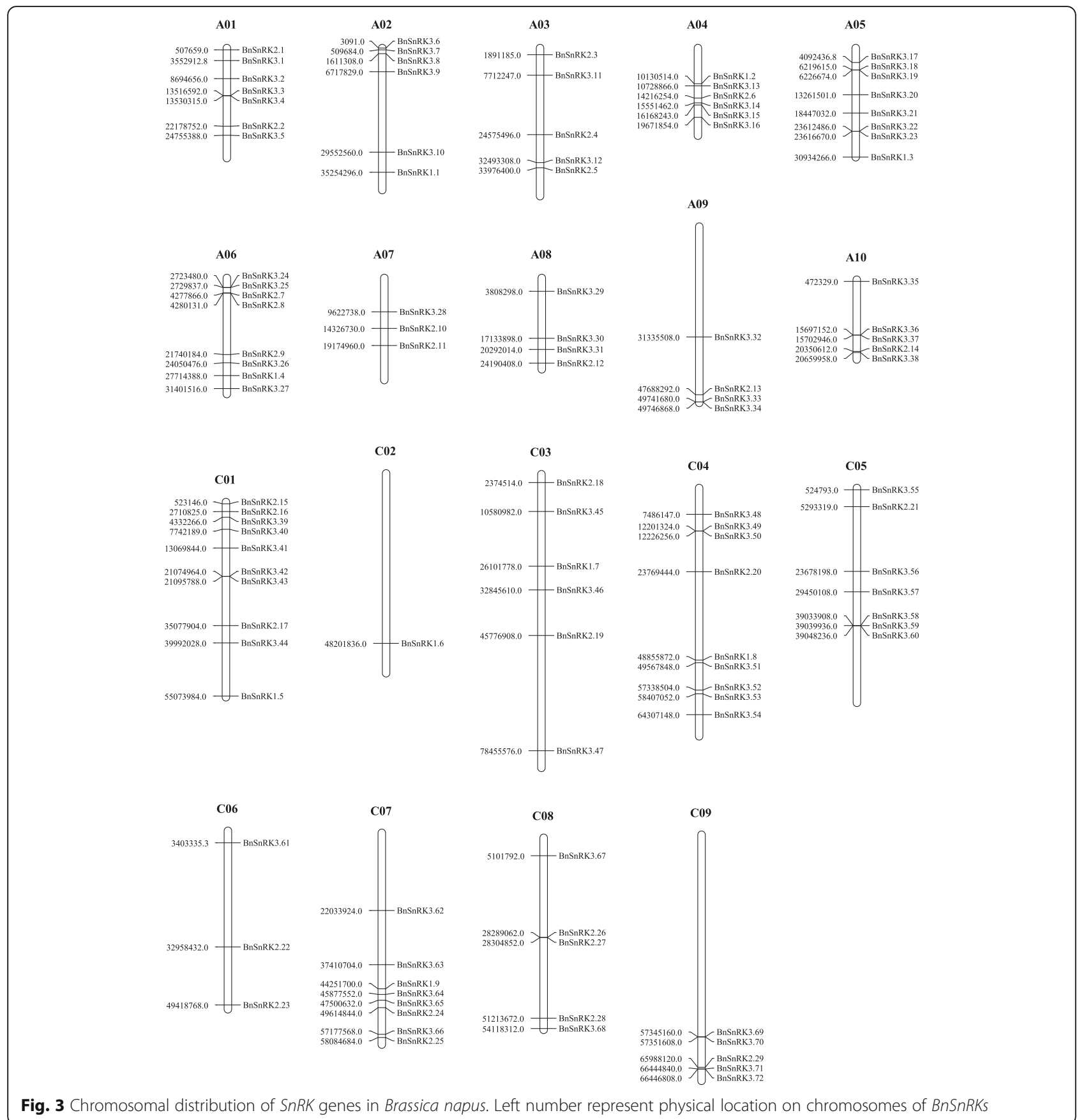

tissues ( $\log 2$-based values $<2)$. Furthermore, 6 genes belonging to the group3 (BnSnRK2.26/2.27 and BnSnRK3.18/3.22/3.43/3.59) were even not expressed in all tissues. Meanwhile, the group1 contained 6 BnSnRK1, $21 B n S n R K 2,40 B n S n R K 3$ genes; the group 2 had 2 $B n S n R K 1,4 B n S n R K 2,15 B n S n R K 3$ genes and the group 3 consisted of 2 BnSnRK1, 6 BnSnRK2, 18 BnSnRK3 genes. These results demonstrated that BnSnRKs displayed diverse expression patterns, and genes within the same subfamily also expressed differently.

\section{Expression profiles of BnSnRKs under different abiotic} stresses

The expression pattern of $B n S n R K$ genes under various abiotic stress were studied using the published transcriptome data of B.napus under drought, salinity, ABA induction and cold stresses [28]. Overall, BnSnRK genes significantly changed the expression level under different abiotic stresses (Fig. 8, Table S8). Multiple BnSnRK genes were significantly induced by diverse treatments. For example, $B n S n R K 2.1$ was extremely induced by all 


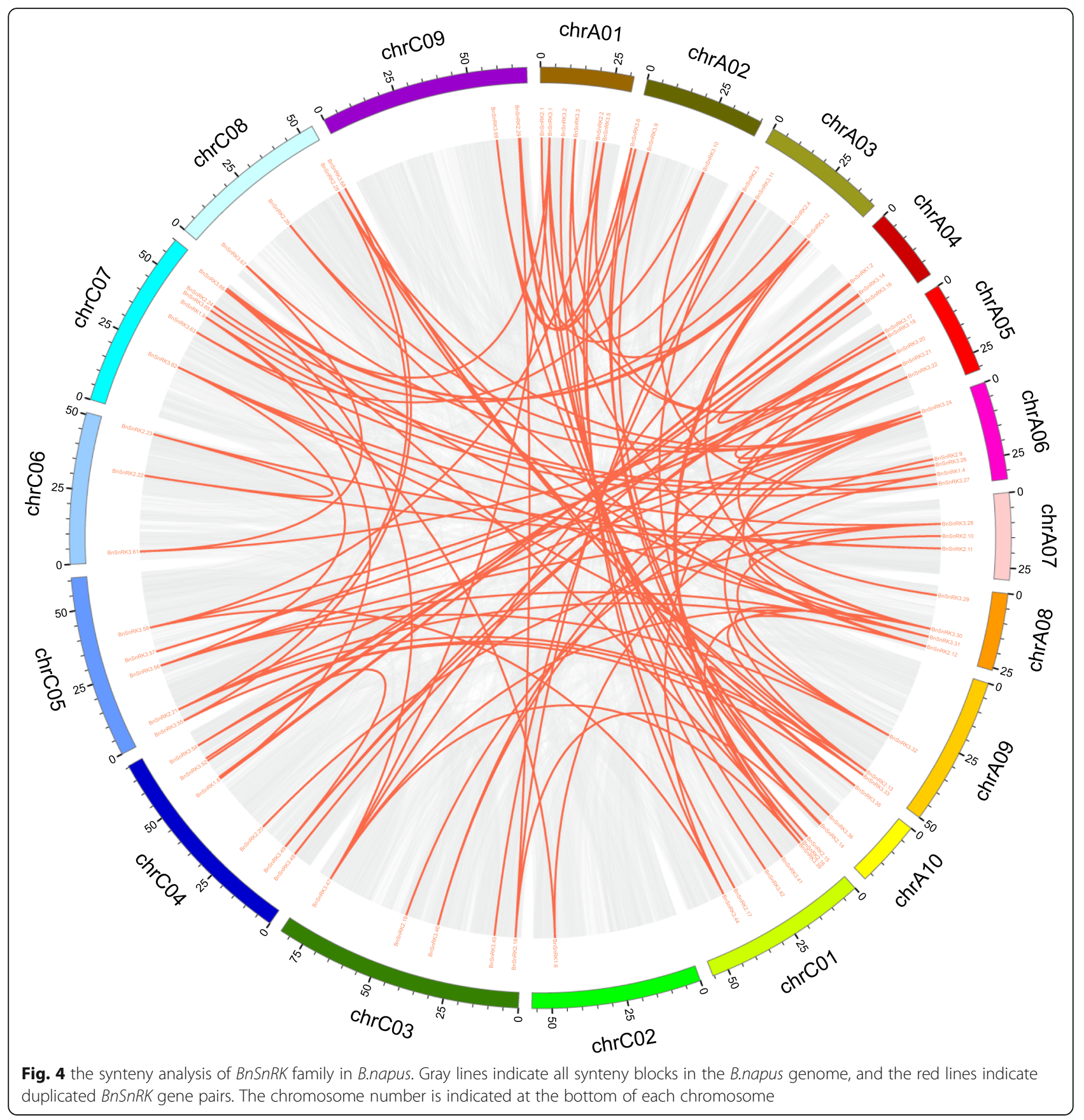

treatments. BnSnRK3.39 incerased expression levels responding to dehydration and ABA treatments. In contrast, several BnSnRKs did not respond to any abiotic stresses. For instance, BnSnRK2.12 and BnSnRK2.13 showed almost no expression changes during all treatments. Interestingly, many genes showed opposing expression profiles under diverse treatments. For instance, BnSnRK3.54 was highly up-regulated by dehydration, whereas was repressed by $\mathrm{ABA}$ and cold treatments.
SNPs analysis of BnSnRKs in a core germplasm of B.napus The polymorphism of $B n S n R K$ genes was determined using our previous resequencing data of a core germplasm resource containing 300 accessions $[29,30]$. The SNPs with MAF more than $5 \%$ in BnSnRK genes were selected out (Table S9). It was shown that each BnSnRK genes contained an average of 11.76 SNPs. In detail, each BnSnRK1 subfamily genes included 14.00 SNPs, each $B n S n R K 2$ and $B n S n R K 3$ subfamily genes contained an average of 9.67 and 12.53 SNPs. Furthermore, the 


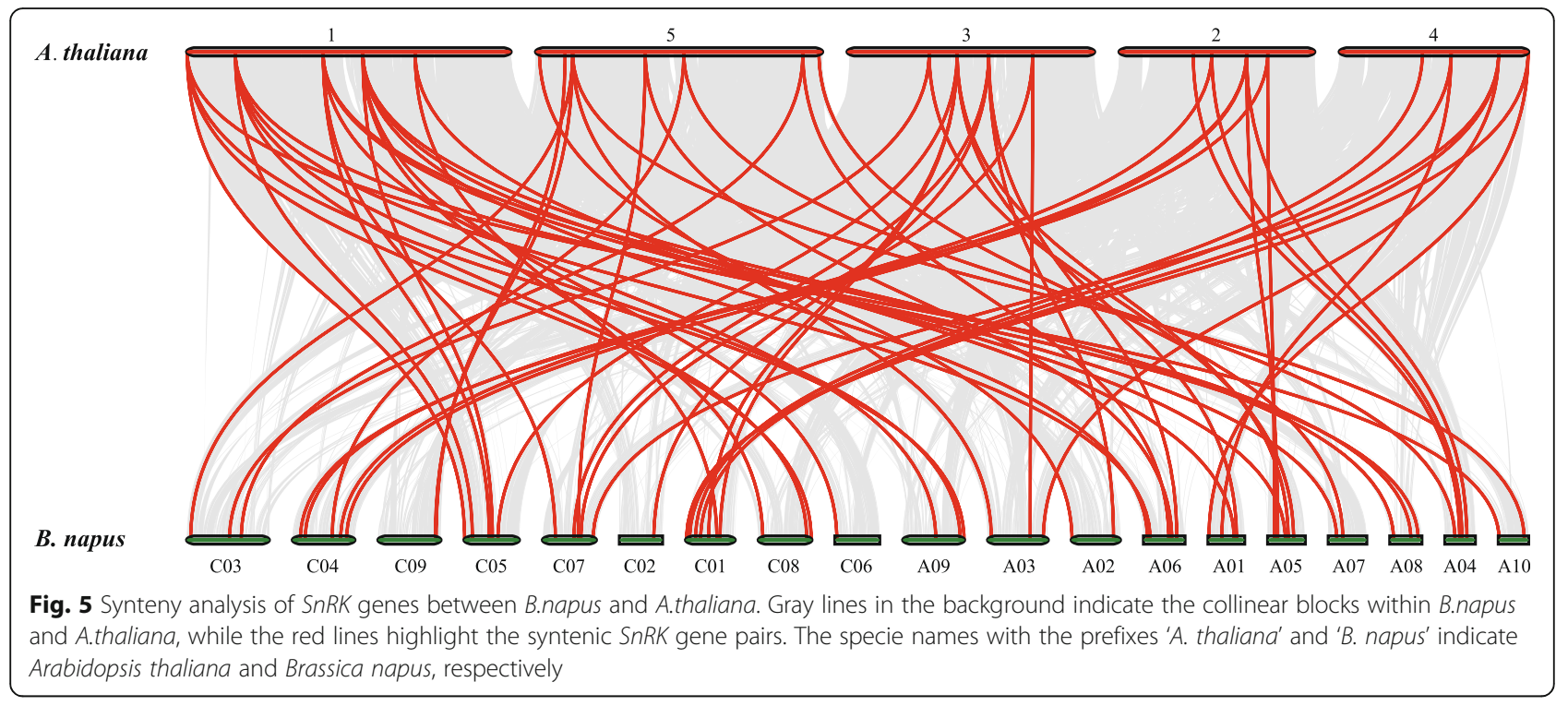

SNP density of each $B n S n R K$ genes within the same subfamily was also different. For instance, there was no SNP identified in BnSnRK1.1, whereas there were 62 SNPs in BnSnRK1.7.

Moreover, a detailed SNP distribution analysis of $B n S n R K 2.10$ and BnSnRK3.39 was conducted because the two genes significantly changed their expression level under drought stress (Fig. 9). It was found that there were 9 SNP loci in the 1500 bp promoter region, 19 SNPs in the exon/intron region and 1 SNP in the 3'UTR region of BnSnRK2.10; while we identified 6 SNP loci in promoter region, $8 \mathrm{SNPs}$ in exon/intron region and 1 SNP in the 3'UTR region of BnSnRK3.39. Combined with phenotypic data of the core germplasm resource under drought stress (data not shown), it was found that SNP16, SNP26, SNP27 in BnSnRK2.10, and SNP4, SNP7, SNP15 in BnSnRK3.39 were significantly associated with their drought tolerance.

\section{Discussion}

In this study, 114 members of $B n S n R K$ genes were identified in the B.napus genome, which were designated as $B n S n R K 1.1$ to BnSnRK3.73 on the basis of their subfamily classification. Systematically investigation on the $B n S n R K$ gene family were carried out, inculding phylogenetic relations, gene structures, protein motifs, chromosome distributions, gene duplication and cis-elements in the promoters. Furthermore, the deep analysis was done on the expression pattern and SNP determination of BnSnRK family genes using published data. This study provides a basic information for further functional characterization of $S n R K$ genes to enhance plant adaptive capacity under abiotic stress.

Previous studies identified 39, 48, 44 and 34 SnRK genes in Arabidopsis thaliana [3], Oryza sativa [31],
Brachypodium distachyon [32] and Eucalyptus grandis [33], respectively. In B.napus genome, BnSnRK gene number is much higher than diplont plants. The 114 $B n S n R K$ genes were identified and classified into three subfamilies, including $10 \mathrm{BnSnRK1}$ genes, $31 \mathrm{BnSnRK2}$ genes and $73 \mathrm{BnSnRK3}$ genes. More specific description showed similar member proportions of each subfamilies between B.napus and other species. B.napus originates from natural crossing of Brapa (AA) and B.oleracea (CC) [25]. 56 and 54 BnSnRK genes were located in the AA subgenome and the $\mathrm{CC}$ subgenome, which indicated that $S n R K$ genes played a similarly important functional role in both ancestral species.

Various SnRK subfamily genes contain different conserved domains, but all genes retained a $\mathrm{N}$-terminal protein kinase domain. For example, SnRK3 subfamily genes were reported interact with CBLs in a calcium-dependent manner because of the NAF domain permits. In addition, the NAF domain defines a set of heterologous kinases involved in diverse signaling processes, as targets of CBL calcium sensor proteins [7]. In this study, it was also found that different BnSnRKs subfamily genes shared the different type of conserved domains. It may suggest there is functional diversification of the $B n S n R K$ gene family according to their domains.

In AtSnRK and BnSnRK gene families, different subfamily genes exhibited significant gene length and exon-intron structural divergences. In previous studies, genes with less introns were considered to have higher expression levels in plants $[34,35]$. A compact gene structure with less introns allowed rapid gene activation and timely response to diverse environmental stresses [35]. However, combined with transcriptome data used in this study, we did not detect that $B n S n R K$ genes with less introns showed higher expression levels than the other BnSnRKs. 


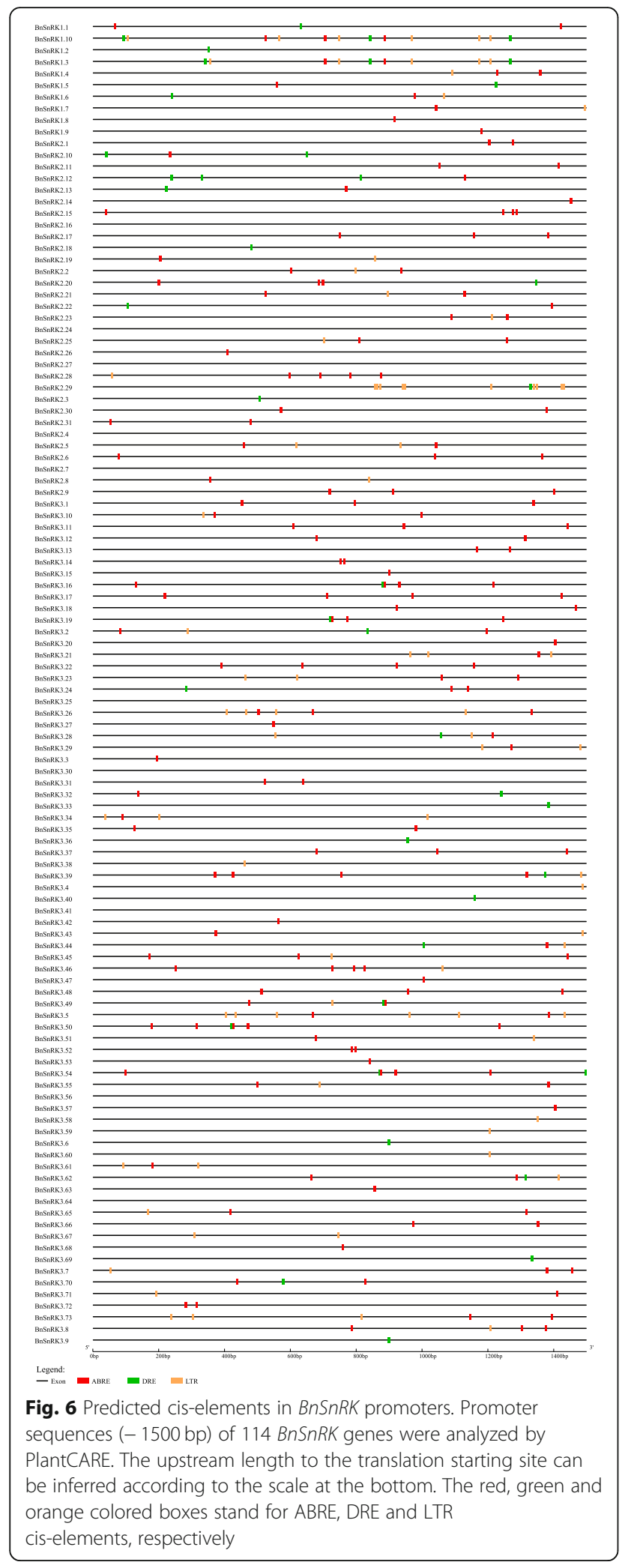

Accumulating evidence suggested that gene activities were generally correlated with disparities in promoter regions [36]. The cis-elements located in the promoter regions of genes played key roles in regulating gene expression during growth and environmental changes $[37,38]$. The promoter analysis revealed that BnSnRKs contained various types of cis-elements, such as DRE, ABRE and LTRE. Most of gene promoters contained at least one of these components, indicating that many of $B n S n R K s$ were able to respond to diverse abiotic stresses. For instance, by combining gene expression profiles under $4 \mathrm{~h}$-cold stress, the expression level of BnSnRKs with LTRE and ABRE cis-elements increased by an average of 2.71-fold, while BnSnRKs with no cis-elements only showed 1.47 -fold increase. Therefore, the cis-elements analysis provide a clue for gene function study, especially for gene expression pattern under different stress.

Gene expression profiles provided imperative clues to map out gene functionality. Firstly, we used prepublished transcriptome data to investigate $B n S n R K$ genes expression levels in diverse tissues and organs of B.napus [26]. The analysis results revealed that the expression patterns of these genes were divided into three groups (Fig. 7). Meanwhile, We found BnSnRKs in group2 contained less cis-elements in promoter than group1 and group3. Specifically, each gene in group1 contains an average of 2.84 ABRE, 0.40 DRE, 0.93 LTRE, and each gene in group3 contains 2.81 ABRE, 0.38 DRE, 0.30 LTRE, whereas each of group2 gene only has 2.00 ABRE, 0.19 DRE, 0.57 LTRE. These evidences suggested that BnSnRKs activities were correlated with disparities in promoter regions.

The roles function of some BnSnRKs reponding to diverse abiotic stresses were also derived. According to the drought stress data (Table S8), the responsive gene $B n S n R K 2.10$ was orthologous to AtSnRK2.3, which regulated $\mathrm{ABA}$ synthesis and signaling responding to drought in A.thaliana [39], indicating identical function of $B n S n R K 2.10$ in responding to drought stress. BnSnRK2.24, exhibited extremely expression changes in drought, salt stresses and ABA induce, while its orthologs AtSnRK2.2, could also respond to osmotic stress and ABA induction in A.thaliana, indicating that BnSnRK2.24 may share similar functions in B.napus [40, 41]. However, BnSnRK3.39 gene, which could be significantly induced under diverse stresses, was only existed in B.napus. Moreover, combined with resequencing data of the core germplasm resource, SNPs of each $B n S n R K$ genes were identified. And the SNP distribution analysis of $B n S n R K s$ such as BnSnRK2.10 and 3.39, could further provide functional molecular markers and alleles for these $B n S n R K$ family genes.

This study provides a comprehensive knowledge of $S n R K$ gene family in B.napus. It gives an important implication for further understanding the biological functions of individual BnSnRK genes in B.napus. However, the study only provided preliminary characterization of 


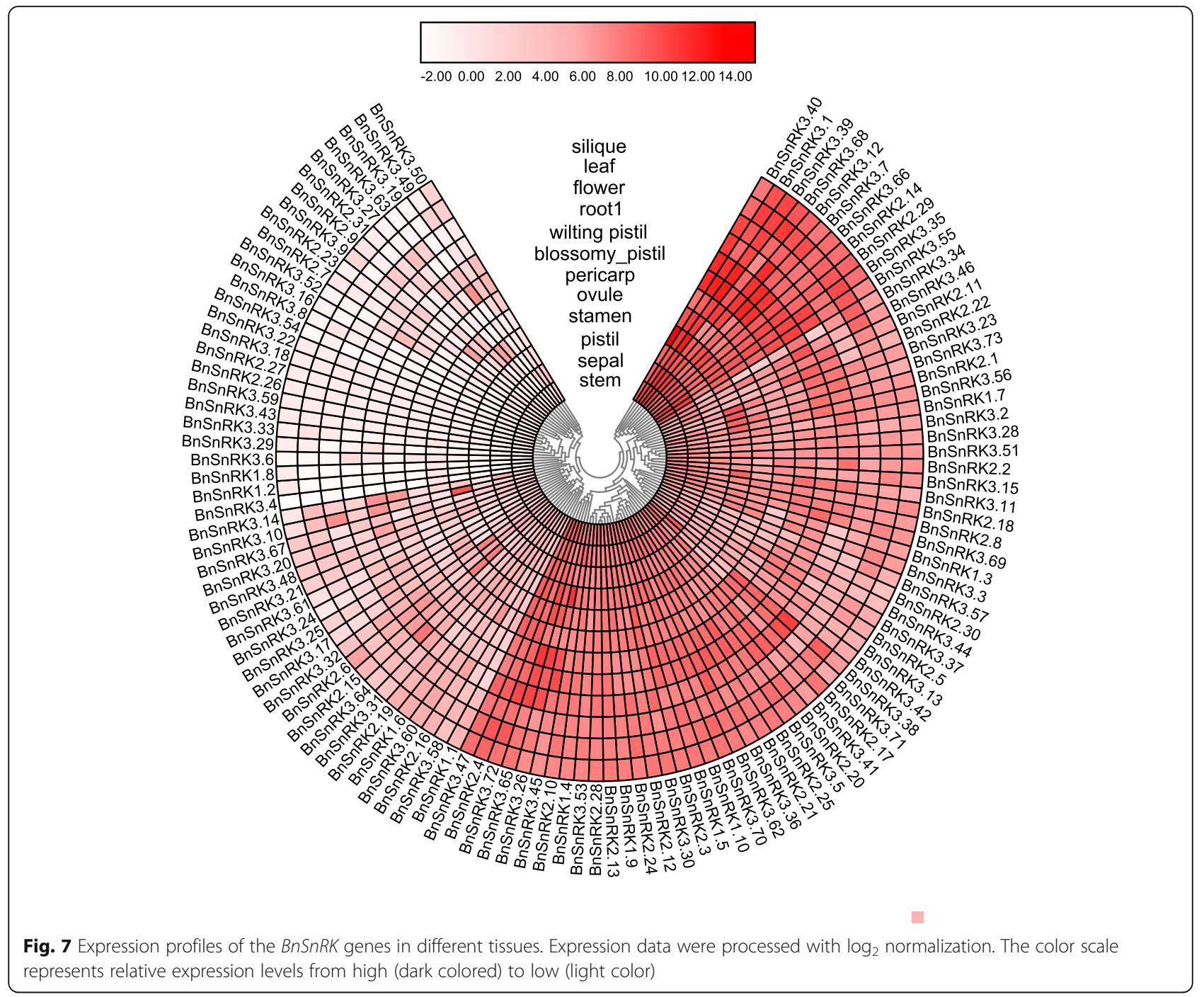

$B n S n R K$ genes and large functional validation work need to be done in further work to understanding the roles of $B n S n R K$ family.

\section{Conclusion}

SnRK genes play important roles in signaling pathways including responses to biotic and abiotic stresses in plants. In this study, a comprehensive study of $S n R K$ gene family in B.napus was performed. A total of 114 $B n S n R K$ genes were characterized and divided into three subfamilies, which showed high similarity in gene structure and motif composition within the same subfamily. Phylogenetic comparison and synteny analysis of SnRK genes between A.thaliana and B.napus provide valuable clues for the evolutionary characteristics of the BnSnRK genes. Moreover, the cis-acting elements, gene expression and SNPs distrubution of $B n S n R K$ family were also determined. These results provide an important information for further understanding biological functions of $B n S n R K$ genes in B.napus.

\section{Methods}

Identification of BnSnRK family genes in the B.napus genome

The amino acid sequences of SnRKs gene family in A.thaliana were downloaded from the NCBI (https://www.ncbi. nlm.nih.gov/). The homologous genes of AtSnRKs in B.napus genome were blasted in the reference genome of rapeseed cultivar Ningyou 7 (http://ibi.zju.edu.cn/bnpedigome/download.php?con=ny7) [27]. Hidden Markov Model (HMM) and BLASTP program were applyed for preliminary identification of BnSnRK proteins. Local BLASTP (E-value-20) searches were performed based on Hidden Markov Model (HMM) profiles of SnRK gene domains from the Pfam database (http://pfam.janelia.org/). All candidate sequences of SnRK genes were reconfirmed through the SMART database (http://smart.embl-heidelberg.de/) [42], the 


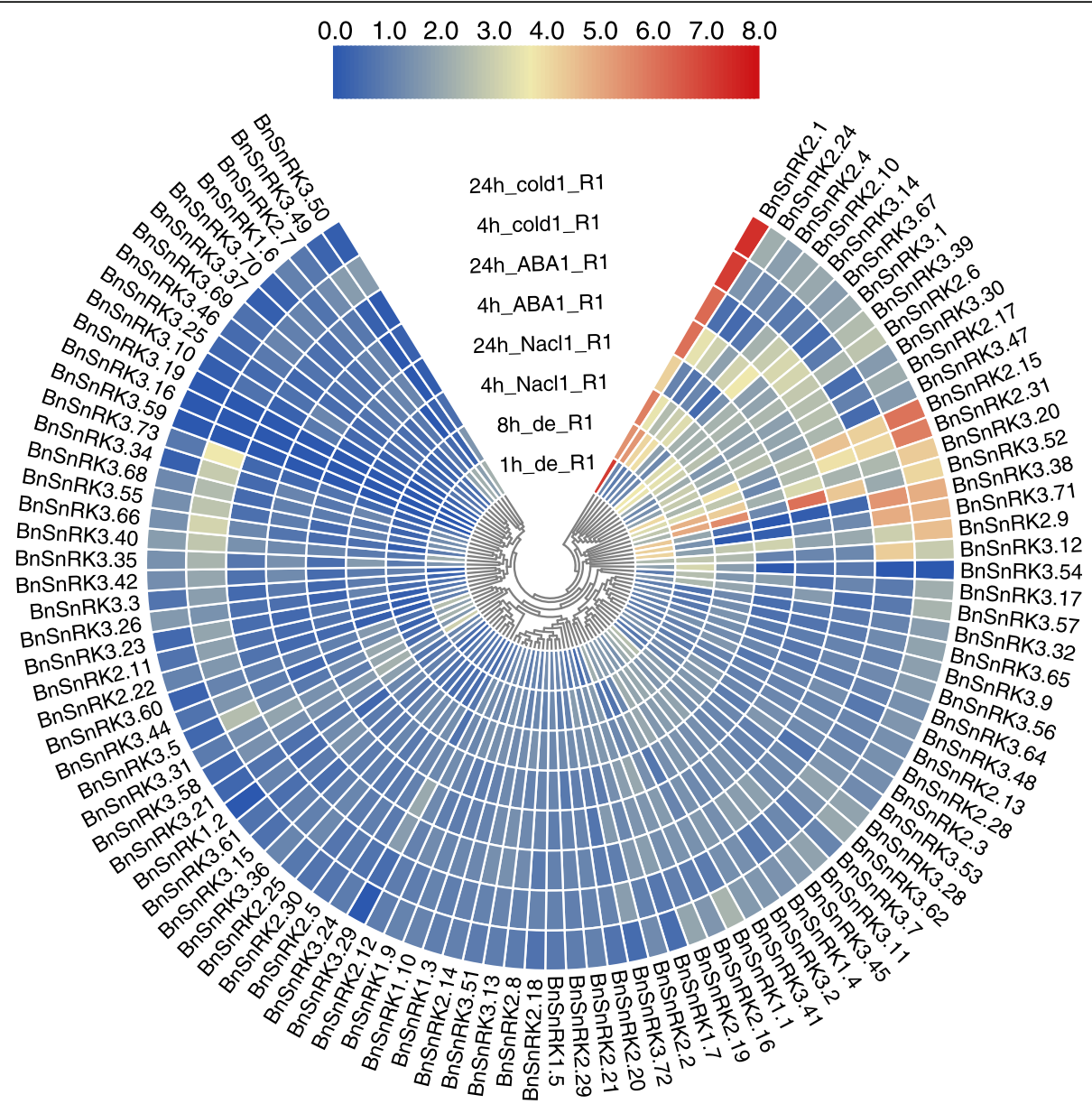

Fig. 8 Expression profiles of the BnSnRK genes under different abiotic stresses. Expression data were the ratio to control values. The color scale represents expression data being processed with $\log _{10}$ normalization. The color scale represents relative expression levels from high (red colored) to low (blue color)

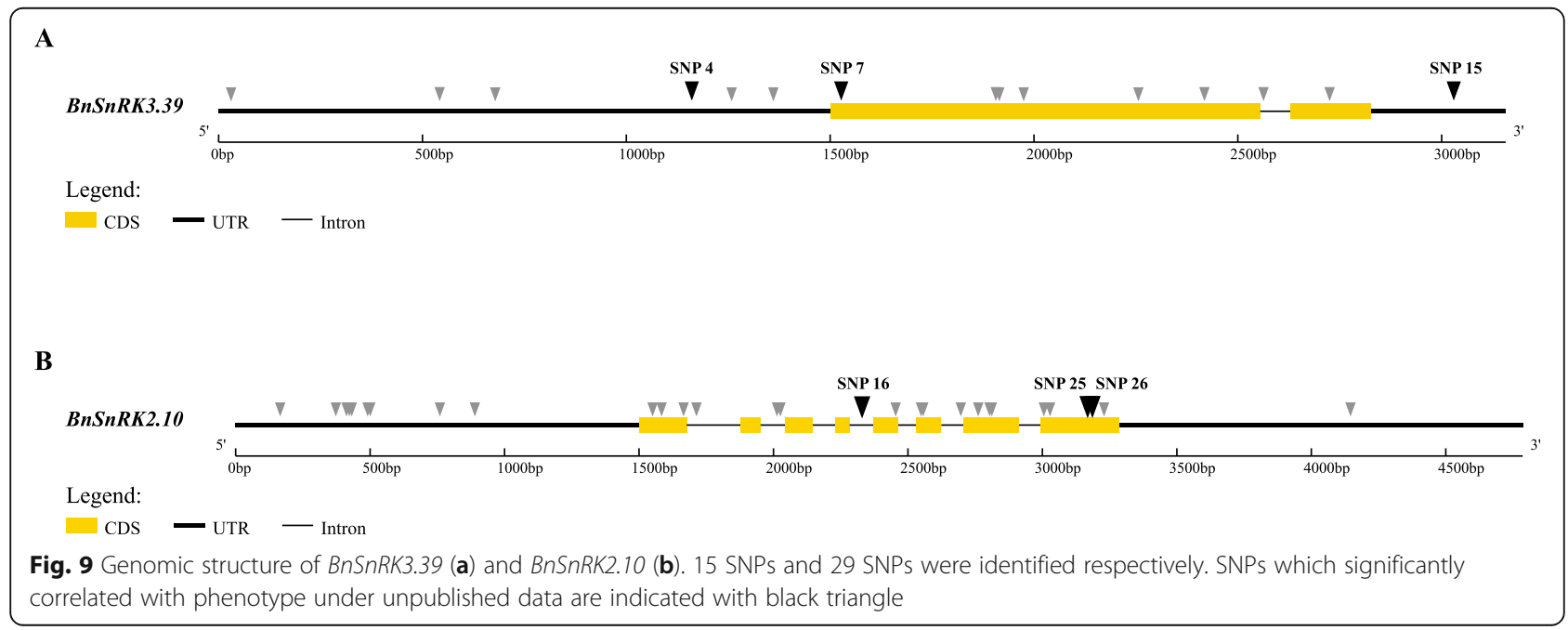


NCBI Conserved Domain database [43] and the Pfam database [44]. Moreover, the number of amino acids, molecular weights $(\mathrm{MW})$ and isoelectric point (pI) of each $S n R K$ protein were calculated using tools from ExPASy (http://www.expasy.ch/tools/pi_tool.html).

\section{Phylogenetic analysis and classification of SnRK gene family in B.napus}

The ClustalW with default parameters was used for multiple sequence alignment of $114 \mathrm{BnSnRK}$ non-redundent amino acid sequences $[45,46]$. Based on the alignments, a phylogenetic tree was constructed using MEGA 7.0 by the Neighbor-Joining (NJ) method [46], with the following parameters: poisson model, pairwise deletion and 1000 bootstrap replications. Unrooted NJ tree of all SnRK protein sequences from A.thaliana and B.napus was also constructed using MEGA 7.0.

\section{Identification of motif compositions and gene structures} To identify conserved motifs of $B n S n R K$ proteins, the Multiple Expectation Maximization for Motif Elicitation (MEME) online program [47] (http://meme.sdsc.edu/ meme/itro.html) was performed with the following parameters: number of repetition $=$ any, maximum number of motifs $=10$; and optimum motif length $=6$ to 100 residues. The exon-intron structures of BnSnRK family genes were analyzed using the Gene Structure Display Server online program (GSDS: http://gsds.cbi.pku.edu.ch) [48].

\section{Chromosomal location and gene duplication}

The chromosomal positions of all BnSnRK genes were mapped to 19 chromosomes of the rapeseed genome according to the physical location information from the NY7 genome database using MapChart version 3.0 and Circos $[49,50]$. To identify gene duplication, all B.napus gene sequences were aligned using BLASTP, with an evalue of 1e-10. MCScanX with default values was used to classify the duplication patterns of the SnRK into segmental, tandem duplications [51]. The definition of tandem duplication is a chromosomal region within $200 \mathrm{~kb}$ containing two or more genes [52]. To exhibit synteny relationships of the orthologous $\operatorname{SnRK}$ genes between B.napus and A.thaliana, the syntenic analysis maps were constructed using python script written by ourselves. Non-synonymous $(K a)$ and synonymous $(K s)$ substitution of each duplicated $B n S n R K$ gene were calculated using KaKs_Calculator 2.0 [53]. Divergence time was estimated using the formula $\mathrm{T}=K s / 2 \mathrm{R}$, where $\mathrm{R}$ is $1.5 \times$ $10-8$ synonymous substitutions per site per year [53].

\section{Cis-elements in promoter regions of BnSnRKs}

Upstream sequences $(1500 \mathrm{bp})$ from the start codon of each BnSnRK gene was extracted from the genome sequence of NY7, and then used for cis-element distributions in promoter regions using PlantCARE software (http://bioinformatics.psb.ugent.be/webtools/plantcare/html/) [54].

\section{Expression patterns of BnSnRKs}

Transcriptome data of 12 different B.napus tissues were obtained from a previous study by Sun et al. [26] under the project ID of PRJNA394926 in the NCBI. Moreover, transcriptome data of B.napus under dehydration, salt, ABA induce and cold stress condition were described in Zhang et al. [28]. The data was available at (https://bigd. big.ac.cn/) under the project ID of CRA001775. Differential expression analysis of BnSnRKs was performed using the DSEeq2 $\mathrm{R}$ package and the heatmaps were created by TBtools software.

\section{SNP distribution analysis of BnSnRKs in B.napus accessions}

The SNPs in the coding regions of $B n S n R K$ genes were extracted from the total SNPs of 300 B.napus accessions, which were determined by the genome re-sequencing in our previous studies $[29,30]$. High-quality SNPs with MAF greater than $5 \%$ and missing rate less than $50 \%$ were used for further analysis. All high-quality SNPs were mapped to the "Darmor-bzh" genome (B.napus v4.1 genome, http://www.genoscope.cns.fr/brassicanapus/data/).

\section{Supplementary information}

Supplementary information accompanies this paper at https://doi.org/10. 1186/s12870-020-02484-3.

Additional file 1: Table S1. The information of $A$. thaliana SnRKs in queries. Table S2. Characteristics of SnRK family in B.napus. Table S3. List of the 114 BnSnRK genes identified in this study. Table S4.

Conserved amino acid motifs and annotation of BnSnRKs. Table S5. Syntenic blocks and Ka/Ks values of BnSnRK genes. Table S6. One-to-one orthologous relationships between B.napus and A.thaliana. Table S7. The expression profiles (log2-based values) of the BnSnRKs under different tissues. Table S8. The expression profiles (ratio to control values) of the BnSnRKs under different abiotic stresses. Table S9. List of the BnSnRK SNPS identified in this study.

\section{Abbreviations}

ABA: Abscisic acid; ABRE: ABA responses; AMPK: AMP-activated protein kinase; AtSnRK: Arabidopsis thaliana SnRK; BnSnRK: Brassica napus SnRK; BLASTP: Basic local alignment search tool-protein; CBLs: Calcium sensor calcineurin B-like proteins; CIPK: CBL-interacting protein kinases;

DRE: Dehydration responses element; GSDS: Gene structure display server; HMM: Hidden markov mode; LTRE: Low-temperature responses element; ROS: Reactive oxygen species; SAPK: Stress-activated protein kinase; SnRK: Sucrose non-fermenting 1 (SNF1)-related protein kinases; SOS: Salt overly sensitive

\section{Acknowledgments}

We thank Dr. Guoping Zhang for his insightful advising and contribution in manuscript revision. 


\section{Authors' contributions}

WZ, DW and LY conceived and designed the research. WZ and LY performed the experiments and data analyses. WZ, DW, $\sqcup$ and LY wrote the article; all authors read and approved the final article.

\section{Funding}

This work was funded by the National Natural Science Foundation of China (31961143008, 31701411), the Science and Technology Program of Zhejiang Province of China (LGN20C130007), Jiangsu Collaborative Innovation Center for Modern Crop Production, and the 111 project for introduction of foreign experts (B17039). None of the funding bodies have any role in the design of the study or collection, analysis, and interpretation of data as well as in writing the manuscript.

\section{Availability of data and materials}

All data analyzed during this study are included in this article and its Additional files.

\section{Ethics approval and consent to participate}

Not applicable.

\section{Consent for publication}

Not applicable.

\section{Competing interests}

The authors declare that they have no competing interests.

\section{Received: 18 February 2020 Accepted: 8 June 2020}

\section{Published online: 22 June 2020}

\section{References}

1. Bohnert HJ, Gong QQ, Li PH, Ma SS. Unraveling abiotic stress tolerance mechanisms-getting genomics going. Curr Opin Plant Biol. 2006;9:180-8.

2. Hunter $\mathrm{H}$. Protein kinases and phosphatases: the yin and yang of protein phosphorylation and signaling. Cell. 1995;80:225-36.

3. Hrabak EM, Chan CW, Gribskov M, Harper JF, Choi JH, Halford N, Kudla J, Luan S, Nimmo HG, Sussman MR, Thomas M, Walker-Simmons K, Zhu JK, Harmon AC. The Arabidopsis CDPK-SnRK superfamily of protein kinases. Plant Physiol. 2003;132:666-80.

4. Halford NG, Hardie DG. SNF1-related protein kinases: global regulators of carbon metabolism in plants? Plant Mol Biol. 1998;37:735-48.

5. Celenza JL, Carlson M. A yeast gene that is essential for release from glucose repression encodes a protein kinase. Science. 1986;233:1175-80.

6. Kulik A, Wawer I, Krzywińska E, Bucholc M, Dobrowolska G. SnRK2 protein kinases-key regulators of plant response to abiotic stresses. OMICS. 2011; 15:859-72.

7. Albrecht $\mathrm{V}$, Ritz O, Linder S, Harter K, Kudla J. The NAF domain defines a novel protein-protein interaction module conserved in $\mathrm{Ca}^{2+}$-regulated kinases. EMBO J. 2011;20:1051-63.

8. Masaru O, Yan Y, Ursula H, Zhu JK. A novel domain in the protein kinase SOS2 mediates interaction with the protein phosphatase 2C ABI2. Proc Natl Acad Sci U S A. 2003;100:11771-6.

9. Hardie DG, Carling D, Carlson M. The AMP-activated/SNF1 protein kinase subfamily: metabolic sensors of the eukaryotic cell? Annu Rev Biochem. 1998;67:821-55.

10. Purcell PC, Smith AM, Halford NG. Antisense expression of a sucrose nonfermenting-1-related protein kinase sequence in potato results in decreased expression of sucrose synthase in tubers and loss of sucrose-inducibility of sucrose synthase transcripts in leaves. Plant J. 1998;14:195-202.

11. Ramon M, Dang TVT, Broeckx T, Hulsmans S, Crepin N, Sheen J, Rolland F. Default activation and nuclear translocation of the plant cellular energy sensor SnRK1 regulate metabolic stress responses and development. Plant Cell. 2019:31:1614-32.

12. Halford NG, Hey SJ. Snf1-related protein kinases (SnRKs) act within an intricate network that links metabolic and stress signalling in plants. Biochem J. 2009;419:247-59.

13. Maszkowska J, Dębski J, Kulik A, Kistowski M, Bucholc M, Lichocka M, Klimecka M, Sztatelman O, Szymanska KP, Dadlez M, Dobrowolska G. Phosphoproteomic analysis reveals that dehydrins ERD10 and ERD14 are phosphorylated by SNF1-related protein kinase 2.10 in response to osmotic stress. Plant Cell Environ. 2019;42:931-46.
14. Diédhiou CJ, Popova OV, Dietz KJ, Golldack D. The SNF1-type serinethreonine protein kinase SAPK4 regulates stress-responsive gene expression in rice. BMC Plant Biol. 2008;8:49.

15. Zhang HY, Jia HF, Liu GS, Yang SN, ZHANG ST, Yang YX, Yang PP, Cui H. Cloning and characterization of SnRK2 subfamily II genes from Nicotiana tabacum. Mol Biol Rep. 2014;41:5701-9.

16. Kawa D, Meyer AJ, Dekker HL, Abd-El-Haliem AM, Gevaert K, Van De Slijke E, Maszkowska J, Bucholc M, Dobrowolska G, De Jaeger G, Schuurink RC, Haring MA, Testerink C. SnRK2 protein kinases and mRNA decapping machinery control root development and response to salt. Plant Physiol. 2020;182:361-77.

17. Anna-Chiara M, Sylvain M, Alain V, Francesca F, Giraudat J. Arabidopsis OST1 protein kinase mediates the regulation of stomatal aperture by abscisic acid and acts upstream of reactive oxygen species production. Plant Cell. 2002;14:3089-99.

18. Ali A, Kim JK, Jan M, Khan HA, Khan IU, Shen MZ, Park J, Lim CJ, Hussain S, Bae D, Wang K, Chung WS, Rubio V, Lee SY, Gong ZZ, Kim WY, Bressan RA, Pardo JM, Yun DJ. Rheostatic control of ABA signaling through HOS15mediated OST1 degradation. Mol Plant. 2019;12:1447-62.

19. Kim KN, Lee JS, Han H, Choi SA, Go SJ, Yoon IS. Isolation and characterization of a novel rice $\mathrm{Ca}^{2+}$-regulated protein kinase gene involved in responses to diverse signals including cold, light, cytokinins, sugars and salts. Plant Mol Biol. 2003;52:1191-202.

20. Guo Y, Xiong L, Song CP, Gong D, Halfter U, Zhu JK. A calcium sensor and its interacting protein kinase are global regulators of abscisic acid signaling in Arabidopsis. Dev Cell. 2002;3:233-44.

21. Liu J, Ishitani M, Halfter U, Kim CS, Zhu JK. The Arabidopsis thaliana SOS2 gene encodes a protein kinase that is required for salt tolerance. Proc Natl Acad Sci U S A. 2000;97:3730-4.

22. Ma QJ, Sun MH, Kang H, Lu J, You CX, Hao YJ. A CIPK protein kinase targets sucrose transporter MdSUT2.2 at Ser (254) for phosphorylation to enhance salt tolerance. Plant Cell Environ. 2019a;42:918-30.

23. Ma QJ, Sun MH, Lu J, Kang H, You CX, Hao YJ. An apple sucrose transporter MdSUT2.2 is a phosphorylation target for protein kinase MdCIPK22 in response to drought. Plant Biotechnol J. 2019b;17:625-37.

24. Chen L, Ren F, Zhou L, Wang QQ, Zhong H, Li XB. The Brassica napus calcineurin B-like 1/CBL-interacting protein kinase 6 (CBL1/CIPK6) component is involved in the plant response to abiotic stress and ABA signalling. J Exp Bot. 2012;63:6211-22.

25. Chalhoub B, Denoeud F, Liu S, Parkin IAP, Tang H, Wang X, Chiquet J, Belcram H, Tong C, Samans B, et al. Early allopolyploid evolution in the post-Neolithic Brassica napus oilseed genome. Science. 2014;345: 950-3.

26. Sun FM, Fan GY, Hu Q, Zhou YM, Guan M, Tong CB, Li JN. The high-quality genome of Brassica napus cultivar 'ZS11' reveals the introgression history in semi-winter morphotype. Plant J. 2017;92:452-68.

27. Zou J, Mao LF, Qiu J, Wang M, Lei J, Wu DY, He ZS, Chen MH, Shen YF, Shen EH, Huang YJ, Li RY, Hu DD, Shi L, Wang K, Zhu QH, Ye CY, lan B, Graham JK, Meng JL, Fan LJ. Genome-wide selection footprints and deleterious variations in young Asian allotetraploid rapeseed. Plant Biotechnol J. 2019;17:1998-2010.

28. Zhang YT, Ali U, Zhang GF, Yu LQ, Fang S, labal S, Li HH, Lu SP, Guo L. Transcriptome analysis reveals genes commonly responding to multiple abiotic stresses in rapeseed. Mol Breed. 2019;39:158.

29. Wu DZ, Liang Z, Yan T, Xu Y, Xuan LJ, Tang J, Zhou G, Lohwasser U, Hua SJ, Wang HY, Chen XY, Wang Q, Zhu L, Maodzeka A, Hussain N, Li ZL, Li XM,

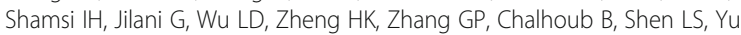
$H$, Jiang $L X$. Whole-genome resequencing of a worldwide collection of rapeseed accessions reveals the genetic basis of ecotype divergence. Mol Plant. 2019;12:30-43.

30. Xuan LJ, Yan T, Lu LZ, Zhao XZ, Wu DZ, Hua SJ, Jiang LX. Genome-wide association study reveals new genes involved in leaf trichome formation in polyploid oilseed rape (Brassica napus L.). Plant Cell Environ. 2019;43:675691.

31. Kobayashi Y, Yamamoto S, Minami H, Kagaya Y, Hattori T. Differential activation of the rice sucrose nonfermenting1-related protein kinase2 family by hyperosmotic stress and abscisic acid. Plant Cell. 2004;16: 1163-77.

32. Wang $L Z$, Hu W, Sun JT, Liang $X Y$, Yang $X Y$, Wei SY, Wang $X T$, Zhou $Y$, Xiao Q, Yang GX, He GY. Genome-wide analysis of SnRK gene family in Brachypodium distachyon and functional characterization of BdSnRK2.9. Plant Sci. 2015;237:33-45. 
33. Wang YJ, Yan HF, Qiu ZF, Hu B, Zeng BS, Zhong CL, Fan CJ. Comprehensive analysis of SnRK gene family and their responses to salt stress in Eucalyptus grandis. Int J Mol Sci. 2019;20:11.

34. Chung BY, Simons C, Firth AE, Brown CM, Hellens RP. Effect of 5'utr intronson gene expression in Arabidopsis thaliana. BMC Genomics. 2006;7:120.

35. Jeffares DC, Penkett CJ, Bähler J. Rapidly regulated genes are intronpoor. Trends Genet. 2008;24:375.

36. Stephen J, Fei N, Juergen E, Zhang Stephen J, Fei N, Juergen E, Zhang S, Dong W, Xue T, Zheng C, Yuan Z. Genome-wide and expression analysis of protein phosphatase 2C in rice and Arabidopsis. BMC Genomics. 2008;9:1-21.

37. Ali GM, Komatsu S. Proteomic analysis of rice leaf sheath during drought stress. J Proteome Res. 2006;5:396-403.

38. Sarda X, Tousch D, Ferrare K, Legrand E, Dupuis J, Casse-Delbart F, Lamaze T. Two TIP-like genes encoding aquaporins are expressed in sunflower guard cells. Plant J. 1997;12:1103-11.

39. Tan WR, Zhang DW, Zhou HP, Zheng T, Yin YH, Lin HH. Transcription factor HAT1 is a substrate of SnRK2.3 kinase and negatively regulates ABA synthesis and signaling in Arabidopsis responding to drought. PLoS Genet. 2018;14:e1007336.

40. Nakashima K, Fujita Y, Kanamori N, Katagiri T, Umezawa T, Kidokoro S, Maruyama K, Yoshida T, Ishiyama K, Kobayashi M, Shinozaki K, YamaguchiShinozaki K. Three Arabidopsis SnRK2 protein kinases, SRK2D/SnRK2.2, SRK2E/SnRK2.6/OST1 and SRK21/SnRK2.3, involved in ABA signaling are essential for the control of seed development and dormancy. Plant Cell Physiol. 2009;50:1345-63.

41. Yoshida T, Fujita Y, Maruyama K, Mogami J, Todaka D, Shinozaki K Yamaguchi-Shinozaki K. Four Arabidopsis AREB/ABF transcription factors function predominantly in gene expression downstream of SnRK2 kinases in abscisic acid signalling in response to osmotic stress. Plant Cell Environ. 2015;38:35-49.

42. Letunic I, Doerks T, Bork P. SMART 7: recent updates to the protein domain annotation resource. Nucleic Acids Res. 2012;40:302-5.

43. Marchler-Bauer A, Lu S, Anderson JB, Chitsaz F, Derbyshire MK, DeWeeseScott C, Fong JH, Geer LY, Geer RC, Gonzales NR, Gwadz M, Hurwitz DI, Jackson JD, Ke Z, Lanczycki CJ, Lu F, Marchler GH, Mullokandov M, Omelchenko MV, Robertson CL, Song JS, Thanki N, Yamashita RA, Zhang D, Zhang N, Zheng C, Bryant SH. CDD: a conserved domain database for the functional annotation of proteins. Nucleic Acids Res. 2011;39:225-9.

44. Finn RD, Coggill P, Eberhardt RY, Eddy SR, Mistry J, Mitchell AL, Potter SC, Punta M, Qureshi M, Sangradorvegas A. The Pfam protein families database: towards a more sustainable future. Nucleic Acids Res. 2016;44:279-85.

45. Saitou N, Nei M. The neighbor-joining method: a new method for reconstructing phylogenetic trees. Mol Biol Evol. 1987;4:406-25.

46. Kumar S, Stecher G, Tamura K. MEGA7: molecular evolutionary genetics analysis version 7.0 for bigger datasets. Mol Biol Evol. 2016;33:1870-4.

47. Bailey TL, Boden M, Buske FA, Frith M, Grant CE, Clementi L, Ren J, Li WW Noble WS. MEME SUITE: tools for motif discovery and searching. Nucleic Acids Res. 2009;37:202-8

48. Hu B, Jin J, Guo AY, Zhang H, Luo J, Gao G. GSDS 2.0: an upgraded gene feature visualization server. Bioinformatics. 2015:31:1296-7.

49. Voorrips RE. MapChart: software for the graphical presentation of linkage maps and QTLs. J Hered. 2002;93:77-8.

50. Krzywinski M, Schein J, Birol I, Connors J, Gascoyne R, Horsman D, Jones SJ, Marra MA. Circos: an information aesthetic for comparative genomics. Genome Res. 2009;19:1639-45.

51. Wang Y, Tang H, DeBarry JD, Tan X, Li J, Wang X, Lee T, Jin H, Marler B, Guo H. MCScanX: a toolkit for detection and evolutionary analysis of gene synteny and collinearity. Nucleic Acids Res. 2012;40:49.

52. Holub EB. The arms race is ancient history in Arabidopsis, the wildflower Nat Rev Genet. 2001;2:516.

53. Wang D, Zhang Z, Zhang Z, Zhu J, Yu J. KaKs Calculator 2.0: a toolkit incorporating gamma-series methods and sliding window strategies. Genomics Proteomics Bioinformatics. 2010;8:77-80.

54. Lescot M, Déhais P, Thijs G, Marchal K, Moreau Y, Yves VDP, Pieree R, Stephane R. Plantcare, a database of plant cis-acting regulatory elements and a portal to tools for in silico analysis of promoter sequences. Nucleic Acids Res. 2002;30:325-7.

\section{Publisher's Note}

Springer Nature remains neutral with regard to jurisdictional claims in published maps and institutional affiliations.

Ready to submit your research? Choose BMC and benefit from:

- fast, convenient online submission

- thorough peer review by experienced researchers in your field

- rapid publication on acceptance

- support for research data, including large and complex data types

- gold Open Access which fosters wider collaboration and increased citations

- maximum visibility for your research: over $100 \mathrm{M}$ website views per year

At $\mathrm{BMC}$, research is always in progress.

Learn more biomedcentral.com/submissions 